\title{
Utility of virtual unipolar electrogram morphologies to detect transverse conduction block and turnaround points of typical atrial flutter
}

\author{
Mitsuru Takami • Akihiro Yoshida • Koji Fukuzawa • Asumi Takei • Gaku Kanda • \\ Kaoru Takami • Hiroyuki Kumagai • Satoko Tanaka • Mitsuaki Itoh • \\ Kimitake Imamura • Ryudo Fujiwara • Atsushi Suzuki • Ken-ichi Hirata
}

Received: 8 January 2011 / Accepted: 21 March 2011 /Published online: 20 April 2011

(C) The Author(s) 2011. This article is published with open access at Springerlink.com

\begin{abstract}
Background Noncontact mapping is useful for the diagnosis of various arrhythmias. Virtual unipolar electrogram morphologies (VUEM) of the conduction block and the turnaround points, however, are not well defined. We compared the VUEM characteristics of a transverse conduction block in the posterior right atrium (RA) with those of contact bipolar electrograms obtained during typical atrial flutter (AFL).

Methods Contact bipolar electrograms were used to map the posterior RA during typical AFL in 16 patients. Twenty points of the VUEM recorded along the block line were analyzed and compared with contact bipolar electrograms. Results Seventeen AFLs were analyzed. Fifteen AFLs showed an incomplete transverse conduction block in the posterior RA by contact bipolar mapping. A double potential on the block line corresponded to the two components of the VUEM, in which the second component showed an Rs, RS, or rS pattern. At the turnaround point, a fused double potential of the contact bipolar electrograms corresponded to a change of the second component of the VUEM from an $\mathrm{rS}$ to a QS morphology. Two AFLs showed a complete block line in the posterior RA. The contact bipolar electrogram showed double potentials from the inferior vena cava to the superior vena cava, whereas the
\end{abstract}

M. Takami $\cdot$ A. Yoshida $(\bowtie) \cdot$ K. Fukuzawa $\cdot$ A. Takei

G. Kanda $\cdot$ K. Takami $\cdot$ H. Kumagai $\cdot$ S. Tanaka $\cdot$ M. Itoh $\cdot$

K. Imamura $\cdot$ R. Fujiwara $\cdot$ A. Suzuki $\cdot$ K.-i. Hirata

Section of Arrhythmia, Division of Cardiovascular Medicine,

Department of Internal Medicine, Kobe University Graduate

School of Medicine,

7-5-2, Kusunoki-Cho, Chuo-Ku, Kobe-shi,

Hyogo 650-0017, Japan

e-mail: ayoshida@med.kobe-u.ac.jp second component of the VUEM remained in an unchanged Rs, RS, or rS pattern.

Conclusion VUEM analysis was a reliable method for identifying the posterior block line during AFL. This method may also be applicable for detecting block lines and turnaround points of circuits in other unmappable arrhythmias.

Keywords Noncontact mapping · Virtual unipolar electrogram - Atrial flutter Conduction block

\section{Background}

Noncontact mapping facilitates radiofrequency ablation of various arrhythmias. The noncontact mapping system simultaneously reconstructs 3,000 virtual unipolar electrograms and superimposes them onto a virtual endocardium, producing isopotential maps with a color-range representing voltage. Compared with electroanatomic CARTO mapping, the noncontact mapping system is especially useful for mapping non-sustained or non-tolerated arrhythmias and shows the reentry circuit around the block line or obstacle by producing a color-coded isopotential map [1, 2] . Visualization of an isopotential map, however, depends to some degree on the color-range setting. Therefore, it is not sufficient to identify the precise length of the block line or the turnaround point of the wavefront using only an isopotential map. The accuracy and reliability of virtual unipolar electrograms around the block line of the reentry circuit, however, are not fully elucidated.

Previous mapping studies in patients with typical atrial flutter (AFL) have described a macroreentrant circuit in the right atrium (RA). For such a circuit to exist, an electrical 
conduction block in the posterior RA is needed to prevent a short circuit between the posterior and lateral walls. Transverse conduction blocks have been evaluated by recording double potentials using contact bipolar electrograms [3-6].

We hypothesized that analysis of the virtual unipolar electrogram morphologies (VUEM) can be used to accurately and reliably detect the location of the conduction block. The purpose of this study was to validate the VUEM characteristics at the block line and the turnaround point and compare them with those of contact bipolar electrograms in the posterior RA during typical AFL.

\section{Methods}

\subsection{Patient characteristics}

This study included 16 patients (mean age $58 \pm 9$ years, 15 men) with typical AFL who underwent an electrophysiologic study and radiofrequency ablation of the cavotricuspid isthmus (CTI). Coexisting paroxysmal atrial fibrillation was documented in five patients. Seven patients had hypertension. None of the patients had other organic heart disease or a history of open heart surgery.

\subsection{Electrophysiologic study}

Informed written consent was obtained from all patients. All antiarrhythmic drugs were discontinued for at least five half-lives before the study. In all patients, venous access was gained through the femoral and subclavian veins to introduce the electrode catheter.

A 5-F 10-polar catheter (2-4-2-mm interelectrode spacing; Boston Scientific, Natick, MA, USA) was inserted into the coronary sinus (CS) with the proximal bipole positioned at the ostium. A 5-F 10-polar catheter (2-5-2-mm interelectrode spacing; St. Jude Medical, St. Paul, MN, USA) was positioned across the tricuspid annulus (TA) to record the His bundle electrogram. A 7-F duodecapolar catheter (Halo, 2-5-2-mm interelectrode spacing; Biosense-Webstar or Livewire, 2-5-2-mm interelectrode spacing, Daig, St. Jude Medical) was positioned along the TA. Care was taken to keep the mapping catheters in the same stable position throughout the study. Pacing output was set to twice the diastolic threshold with a pulse width of $2 \mathrm{~ms}$ using a programmable stimulator (Cardiac Stimulator SEC-3,102; Nihon Kohden, Tokyo, Japan). Bipolar electrograms were recorded using a separate digital system (Prucka Engineering, Houston, TX, USA) with filtering at 30 to $500 \mathrm{~Hz}$. Contact mapping of the RA was performed using a 7-F steerable catheter with a 4-mm distal tip electrode and 2-mm ring electrode with an interelectrode distance of $4 \mathrm{~mm}$ (Japan Lifeline, Tokyo, Japan) (Fig. 1(a), (b)).

\subsection{Noncontact mapping system}

The noncontact mapping system (EnSite3000, Endocardial Solutions Inc., St. Paul, MN, USA) was previously described in detail [1]. The system comprises a noncontact catheter $(9 \mathrm{~F})$ with a multielectrode array (MEA) surrounding a 7.5-ml balloon mounted at the distal end. Raw data detected by the MEA are transferred to a Silicon Graphics workstation via a digitalized amplifier system.

By dragging and rotating the conventional catheter with the locate signal around the RA, the noncontact mapping system receives a sample of geometric points, allowing for the construction of a three-dimensional (3D) computer model of the RA endocardium. The distance between the endocardial surface and the recording electrode can be used to compute the filtered unipolar far-field ECGs from the 64 wires of the MEA catheter for up to 3,360 endocardial electrograms over the entire RA endocardium based on an inverse solution of Laplace's equation. The calculated electrograms are displayed on the Silicon Graphics workstation as conventional electrograms at any chosen endocardial point or time as a color-coded isopotential map.

Before deployment of the MEA, patients were given 3,000 IU heparin boluses to maintain activated clotting time over $250 \mathrm{~s}$ throughout the study. The MEA catheter was deployed over a 0.035-in. guide wire, which was advanced to the superior vena cava (SVC). In the RA, the balloon was inflated with a saline-contrast mixture. The locator signal was passed between the ring electrodes of the MEA catheter and the tip electrode of the mapping catheter. The mapping catheter was placed at the CS orifice, TA, and the borders between the RA and the SVC and inferior vena cava (IVC), and the location of these landmarks was tagged on the virtual endocardium of the EnSite system. Virtual unipolar electrograms were displayed after filtering through a bandpass filter between 2 and $150 \mathrm{~Hz}$.

If spontaneous AFL was found at the onset of the study, both noncontact and contact mapping were performed simultaneously to investigate the posterior block line. If sinus rhythm was present at baseline, atrial burst pacing was performed to induce AFL.

After the 3D geometry of the RA was constructed, 20 points of virtual unipolar electrograms were recorded along the posterior block line from the IVC to the SVC during AFL (Fig. 1(c)), and contact bipolar mapping was simultaneously performed on the same points.

\subsection{Entrainment mapping}

Entrainment pacing of 10 or $20 \mathrm{~ms}$ below the cycle length of the AFL was performed from the CTI and 3 points around the posterior block line (septal side, lateral side, and turnaround point) during AFL and the postpacing interval 


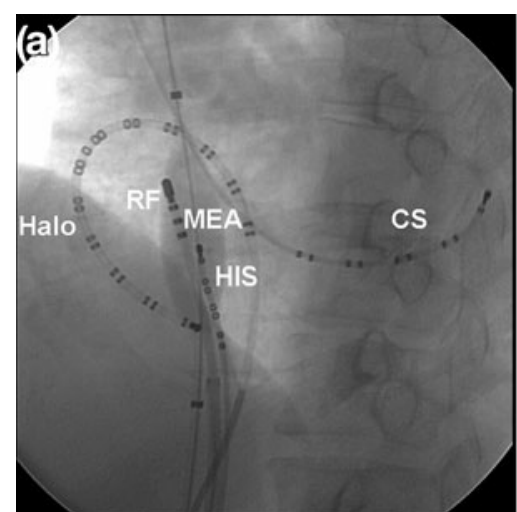

Fig. 1 (a, b) Fluoroscopic view of noncontact balloon and other catheter in the right atrium $(R A)$ during contact mapping of the posterior RA. (a) $45^{\circ}$ left anterior oblique and (b) $30^{\circ}$ right anterior oblique. $C S$ coronary sinus, $M E A$ multielectrode array, HIS catheter recording the His bundle electrogram, Halo Halo catheter spans the tricuspid annulus,
$R F$ mapping and ablation catheter. (c) Geometry of the right atrium (posterior-anterior view: PA). (※) 20 points of virtual unipolar electrograms were recorded in the posterior right atrium. $S V C-R A$ junction junction between the superior vena cava and the RA, IVC-RA junction junction between the inferior vena cava and the RA
(PPI) was measured. If the difference between the PPI and the AFL cycle length was less than $30 \mathrm{~ms}$, the site was considered to be within the circuit.

\subsection{Ablation and recognition of bidirectional isthmus block}

CTI linear ablation was performed using an Ablaze 8-mm tip electrode catheter (Japan Lifeline, Tokyo, Japan) with a maximal target temperature of $60^{\circ} \mathrm{C}$ and a maximum delivered power of $40 \mathrm{~W}$. The endpoint of AFL ablation was the bidirectional block of CTI. The complete clockwise isthmus block pattern was defined as a purely craniocaudal atrial activation sequence along the Halo catheter during proximal CS pacing. The distal bipole of the Halo catheter should be depolarized later than the more proximal bipoles. A complete counterclockwise isthmus block pattern was defined as depolarization of the proximal CS area recorded later than that at the bundle of His region during low lateral RA pacing. In addition, we confirmed the bidirectional block using the differential pacing criterion [7].

\subsection{Definition}

Typical AFL (counterclockwise rotation: CCW) and reverse typical AFL (clockwise rotation: $\mathrm{CW}$ ) were confirmed based on the following criteria: characteristic surface ECG morphology, counterclockwise or clockwise activation sequence around the TA on intracardiac electrography, concealed entrainment from the CTI, and arrhythmia termination during CTI ablation.

The point of the border between the SVC and RA was tagged at the base of the RA appendage using a biplane fluoroscopy image and the 3D geometry of the EnSite system. The posterior border between the SVC and RA was defined as the point on the virtual endocardium at the same level of the anterior point. In the same way, the border between the RA and IVC was defined as the inflection point of CTI from the RA to the IVC, based on the right anterior oblique fluoroscopy image and $3 \mathrm{D}$ geometry. The length of the posterior RA was defined as the distance between the borders of the SVC and IVC.

The block line of the posterior RA was detected by the double potentials of contact bipolar electrograms, characterized by two discrete deflections separated by an isoelectric or low amplitude line [8]. The upper limit of the block line where the wavefront of the AFL turned around was defined as the point of the fused double potentials or fragmented potential of contact bipolar electrograms.

\subsection{Virtual unipolar electrogram morphologies}

The "rS" morphology was defined as one small positive deflection followed by a large negative deflection with $\mathrm{r} / \mathrm{S}<1$. The "Rs" morphology was defined as one large positive deflection followed by a small negative deflection with $\mathrm{R} / \mathrm{s}>1$. The "RS" morphology was defined as one positive deflection followed by a negative deflection with $\mathrm{R} / \mathrm{S}=1$. The "QS" morphology was defined as only one large negative deflection. The " $R$ " morphology was defined as only one large positive deflection.

\section{Results}

\subsection{Patients}

AFL was present in 12 patients at the beginning of the session. In the other four patients, AFL could be induced by programmed stimulation. 
Seventeen isthmus-dependent AFL were observed in 16 patients. Typical AFL (CCW) was observed in 12 patients, reverse typical AFL $(\mathrm{CW})$ in 3 patients, and both typical and reverse typical AFL in 1 patient. Mean cycle length of AFL was $233 \pm 26 \mathrm{~ms}$. The mean distance from the 20 points of the virtual unipolar electrograms to the MEA center was $24.5 \pm 2.2 \mathrm{~mm}$.

\subsection{Incomplete transverse conduction block}

In 15 AFL (88\%, CCW: $n=13, \mathrm{CW}: n=2)$, the block line did not extend from the IVC to the SVC. The double potentials of contact bipolar electrograms recorded at the lower part of the posterior RA changed to a fused double potential or fragmented potential at the upper part of the posterior RA. The fused double potential or fragmented potential indicates the turnaround point of the wavefront, $i$. e., the upper limit of the block line (Fig. 2(a)). The colorcoded isopotential maps of the noncontact mapping also revealed that the activation wavefront of the posterior RA during typical AFL went up on the septal side, crossed the gap between the SVC and the posterior block line, and propagated downward on the free wall side (Fig. 2(b)). The wavefront propagated in the opposite direction from the septal to free wall side during reverse typical AFL.

The VUEM along the block line were divided into three morphologic patterns.

$\square$ At the lower part of the block line, the VUEM presented two components. The second component was an Rs, RS, or rS pattern.

$\square$ At the turnaround point of the wavefront, i.e., the upper limit of the block line, the VUEM also showed two components, but the second component was changed from rS to QS in all 15 AFL.

$\square$ At the area between the upper limit of block line and the SVC orifice, the VUEM demonstrated two patterns. One pattern had two components, with the second component being QS. Another pattern had a single component, either Rs, RS, or rS (Figs. 2(a) and 4(a)).

\subsection{Complete transverse conduction block}

In two AFL (12\%, CW: $n=2)$, the block line extended to the SVC. The double potentials of contact bipolar electrograms were continuously recorded from the IVC to the SVC at the posterior RA. The color-coded isopotential maps also demonstrated that the activation wavefront did not cross the posterior RA from the free wall to the septal side (Fig. 3(a)).

The VUEM showed two components continuously along the block line. The second component maintained an Rs, $\mathrm{RS}$, or $\mathrm{rS}$ pattern from the IVC to the SVC and did not change to a QS pattern in each of the two AFL cases (Figs. 3(b) and 4(b)).

\subsection{Entrainment mapping}

Entrainment mapping was performed during $13 \mathrm{AFL}$ (CCW: $n=11, \mathrm{CW}: n=2$ ). The PPI minus cycle length (CL) of AFL was less than $30 \mathrm{~ms}$ at the cavotricuspid isthmus in all of the AFL. When pacing from the three points around the posterior block line (septal side of the block line, lateral side of the block line, and the point between the upper limit of the block line and posterior SVC), the PPI minus CL of AFL was also less than $30 \mathrm{~ms}$ at all three points during AFL (Fig. 5).

\subsection{Ablation}

A bidirectional TA-IVC isthmus block was achieved in all patients and none of the patients experienced an AFL recurrence during a mean follow-up of $360 \pm 262$ days.

\section{Discussion}

\subsection{Major findings}

This study demonstrated the reliability of VUEM at the turnaround point of the wavefront during typical and reverse typical AFL. In cases with an incomplete transverse conduction block, the second component of the VUEM changed from an $\mathrm{rS}$ to a QS pattern at the upper limit of the block line. In cases with a complete transverse conduction block, the second component of the VUEM maintained a continuous Rs, RS, or rS pattern along the posterior block line. The circuits around the posterior block line were confirmed within the reentry circuit by entrainment pacing.

A transverse conduction block at the posterior RA is essential for the perpetuation of typical AFL. Previous studies reported that the transverse conduction blocks were able to be evaluated by recording double potentials using contact bipolar electrograms [3-6]. Noncontact mapping systems facilitate the identification of the circuits of reentrant arrhythmias $[9,10]$. In particular, the isopotential map is useful for detecting the block line and gaps in the block line during reentrant arrhythmias. Zheng et al. [11] reported that noncontact mapping could identify the gap accurately and quickly to target the effective ablation site during stepwise linear ablation for atrial fibrillation. Visualization of an isopotential map, however, depends to some degree on the color-range setting. Therefore, it is not sufficient to identify the precise length of the block line or the turnaround point of the wavefront using only an isopotential map. In the present study, we tried to evaluate 
Fig. 2 Incomplete block line during typical AFL (37-yearold female; CL $210 \mathrm{~ms}$,

counterclockwise rotation). (a) Comparison of virtual unipolar electrogram morphologies (VUEM) and contact bipolar electrograms. The block line did not extend to the superior vena cava $(S V C)$. The double potentials of contact bipolar electrograms recorded at the lower part of the posterior right atrium $(R A)$ changed to a fused double potential at the upper part of the posterior RA. The fused double potential of contact bipolar electrograms (upper limit of the block line) is shown on the geometry map. The VUEM showed two components on the block line, and the second component of the VUEM was either an Rs or rS pattern on the block line. At the upper limit of the block line, the second component of the VUEM changed from an $\mathrm{rS}$ to a QS pattern. (b) Color-coded isopotential map of RA geometry. The activation wavefront goes up the septal side (frames 1-2) and crosses the posterior RA (frames 3-4) and propagates downwards to the lateral side (frames 5-6). $P A$ view posterior-anterior view, $L A O$ view left anterior oblique view, $S V C$ superior vena cava, $I V C$ inferior vena cava
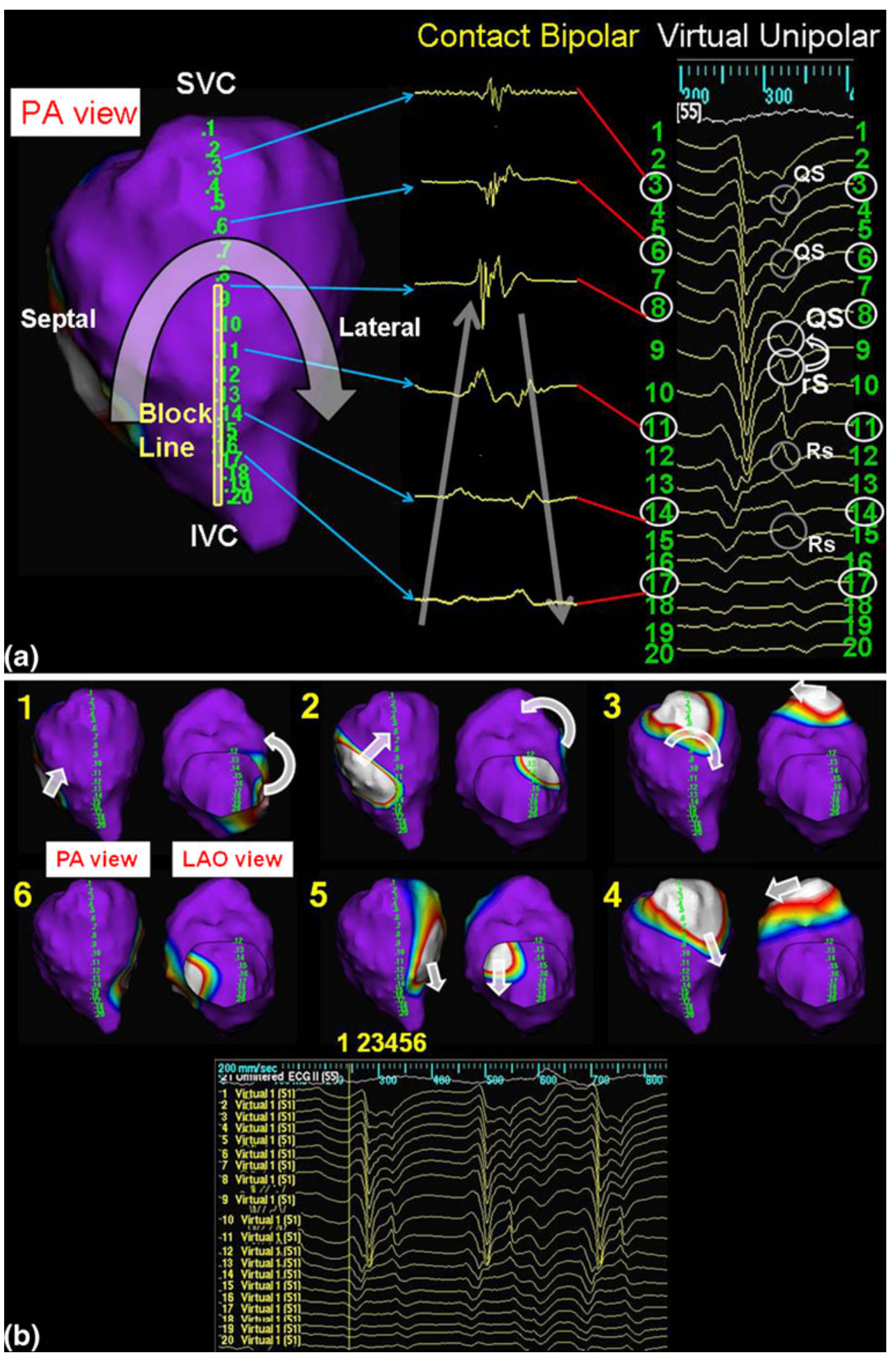

the new characteristics of the VUEM at the block line and turnaround point compared with the conventional contact bipolar electrograms in the posterior RA during AFL. We demonstrated that the specific change in the second component of the VUEM could indicate the turnaround point of the wavefront independent of the color-range setting.

\subsection{Previous studies of unipolar electrograms}

Spach et al. [12, 13] demonstrated that the conduction along the longitudinal axis of the myocardial fibers produces RS morphologies of contact unipolar electrograms. The positive uniphasic unipolar $\mathrm{R}$ electrograms are characteristic of the end of the propagation. The negative 
Fig. 3 Complete block line during reverse typical AFL (62-year-old male; CL 275 ms, clockwise rotation). a Comparison of virtual unipolar electrogram morphologies (VUEM) and contact bipolar electrograms The block line extended to the superior vena cava $(S V C)$ orifice. Double potentials of contact bipolar electrograms were continuously recorded from the inferior vena cava to the SVC at the posterior right atrium $(R A)$. The VUEM showed two components on the block line, and the second component of the VUEM remained unchanged in an $\mathrm{rS}$ or Rs pattern. (b) Color-coded isopotential map of the RA geometry. The activation wavefront goes up the lateral side (frames 1-2), but cannot cross the posterior RA (frames 3). The wavefront propagates to the anterior SVC (frames 4) and downward to the septal side (frames 5-6). PA view posterior-anterior view, $L A O$ view left anterior oblique view, $S V C$ superior vena cava, $I V C$ inferior vena cava
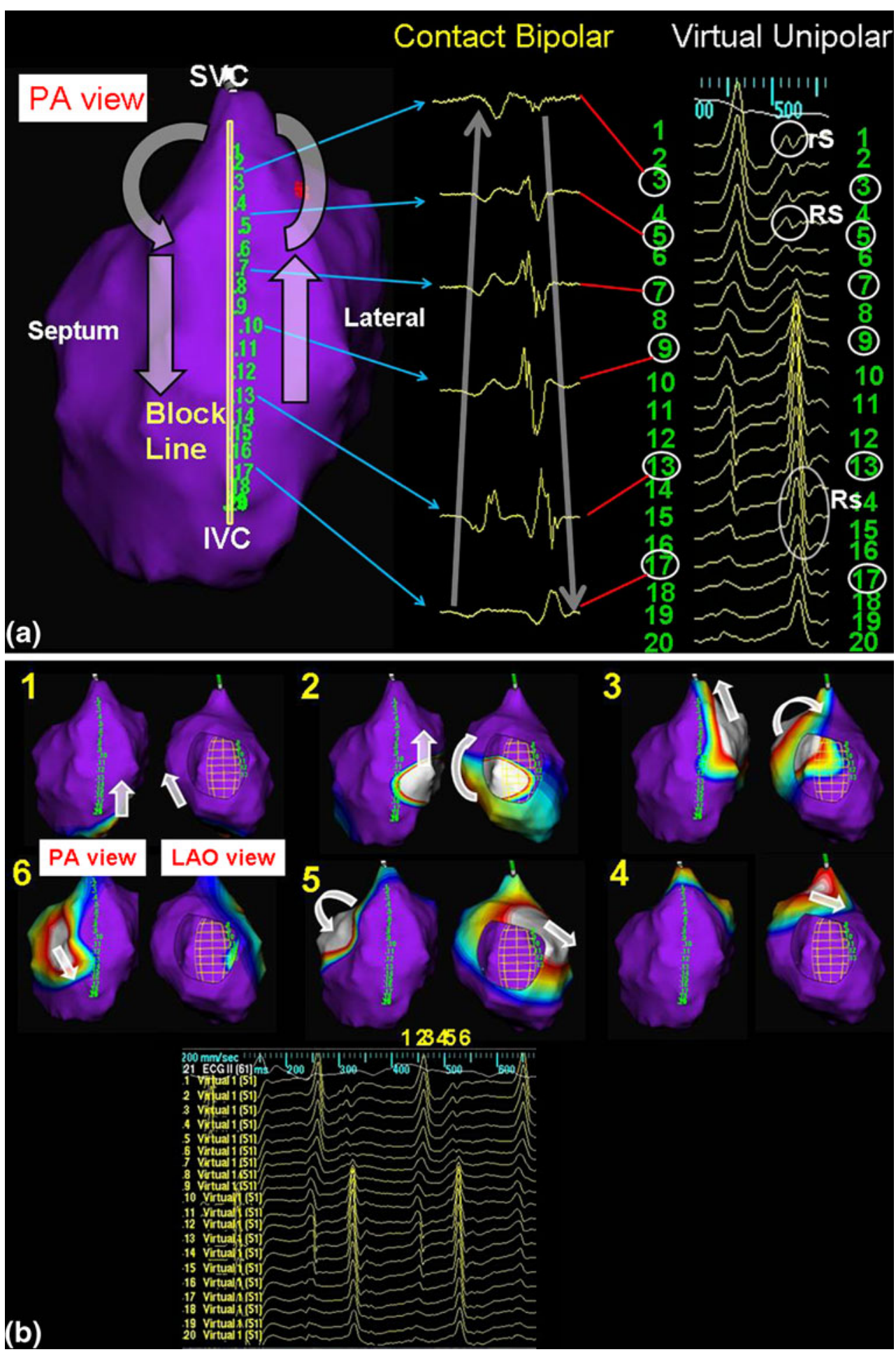

QS waveforms are recorded in the vicinity of the site of the excitation onset. Villacastin et al. [14] reported the usefulness of recording contact unipolar electrograms to assess the conduction of the cavotricuspid isthmus during radiofrequency ablation of typical AFL. They analyzed the morphologies of contact unipolar electrograms of the ablation line before and after the first procedure. During pacing on one side of the cavotricuspid isthmus, the contact unipolar electrogram on the opposite side showed an RS, rS, or QS pattern before ablation, which changed to an R or Rs pattern after achieving a complete cavotricuspid isthmus block.

On the other hand, Lin et al. [15] investigated the characteristics of the second component of the VUEM 
(a)

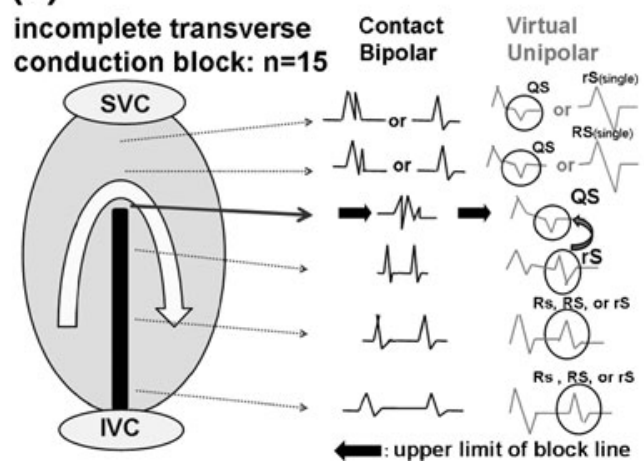

Fig. 4 Schema of a virtual unipolar electrogram morphology (VUEM) pattern. (a) Incomplete transverse conduction block $(n=15)$ : The wavefront crossed the gap between upper limit of the block line and the superior vena cava $(S V C)$. On the block line, contact bipolar electrograms showed double potentials. The VUEM had two components and the second component had either an Rs, RS, or rS pattern. At the turnaround point of the wavefront (upper limit of the block line), a fused double potential or fragmented potential was recorded in contact bipolar electrograms. The second component of the VUEM changed from $\mathrm{rS}$ to QS at the upper limit in all cases of

recorded at the cavotricuspid isthmus after ablation in patients with typical AFL using a noncontact mapping system. They reported that the second component of the VUEM showed an $\mathrm{R}$ or Rs pattern at a complete cavotricuspid isthmus block and an $\mathrm{rS}$ pattern at an incomplete cavotricuspid isthmus block, suggesting that VUEM is useful for detecting whether the conduction block is complete. Although these previous studies demonstrated the usefulness of unipolar morphologies, the ability to determine the turnaround point of the wavefront or the upper limit of the block line using VUEM was not elucidated.

\subsection{VUEM at the block line and the turnaround point} of the wavefront

In this study, 20 points of VUEM in the posterior RA were analyzed to detect the block line and the turnaround point of the wavefront. The results provided evidence that the second component of the VUEM shows an Rs, RS, or rS pattern at the transverse conduction block, which changes to a QS pattern at the turnaround point of the wavefront. The wavefront of typical AFL goes up on the septal side of the block line and propagates downward on the free wall side. The first component of the VUEM reflects the upward propagation and the second component reflects the downward propagation. Therefore, the second component of the VUEM showed an Rs, RS, or rS pattern at the block line representing the wavefront coming in the craniocaudal direction. At the upper limit of the block line, the second component of the VUEM changed from rS to QS pattern in this study.

\section{(b)}

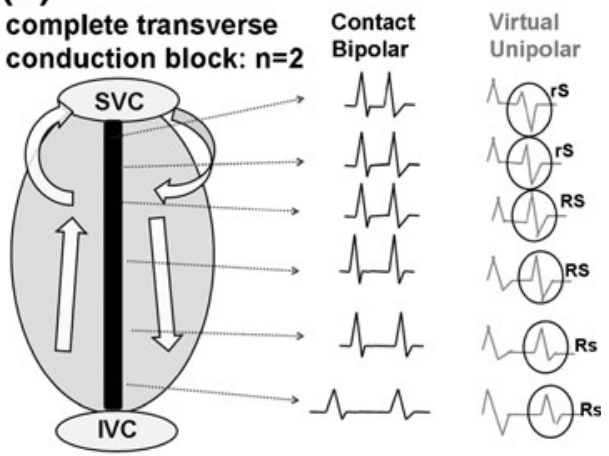

atrial flutter. At the area between the upper limit of the block line and the SVC orifice (transversal conduction gap), the VUEM showed two patterns. In one pattern, the second component of the VUEM was a QS, and in the second pattern, the component was a single potential (Rs, RS, or rS). (b) Complete transverse conduction block $(n=2)$ : Contact bipolar electrograms showed double potentials from the IVC to the SVC at the posterior RA, and the second component of VUEM remained an unchanged $\mathrm{rS}$ or RS or Rs pattern along the block line. $S V C$ superior vena cava, $I V C$ inferior vena cava

We speculate that these changes of unipolar morphologies are caused by the very slow transverse conduction of the wavefront at the pivot point, i.e., the upper limit of the block line. The non-uniform anisotropic muscular pattern was shown in the junctional area of the crista terminalis with the pectinate muscle and with the sinus venosa region in the RA [16]. At the pivot points, the curvature of the circulating wave front is high in anisotropic myocardium. The excitatory current is dissipated, and consequently, a curved wave front propagates at reduced speed because a convex wave front must excite more tissue ahead than a planar wave front [17]. The curvature effect of wavefront may cause slowing of propagation and conduction block. In fact, the contact bipolar showed the fused double potentials or fragmented potential at the pivot point in this study, which means the slow conduction at this point. The first component of VUEM reflects that the wavefront comes to this point, and the electric line between the two components is caused by the very slow conduction at this point. The second component of VUEM showed a QS pattern because the propagation spread from this point to the opposite side.

\subsection{Upper turnaround portion of the circuit of AFL}

A transverse conduction block at the posterior RA is essential for the perpetuation of typical AFL. Fukuzawa et al. [4], however, reported that the transverse conduction in the posterior RA is not complete and that the wavefront turns around at the posterior RA below the SVC in the majority of AFL patients, based on the CARTO mapping system. Dixit et al. [18] reported the evidence of posterior RA 


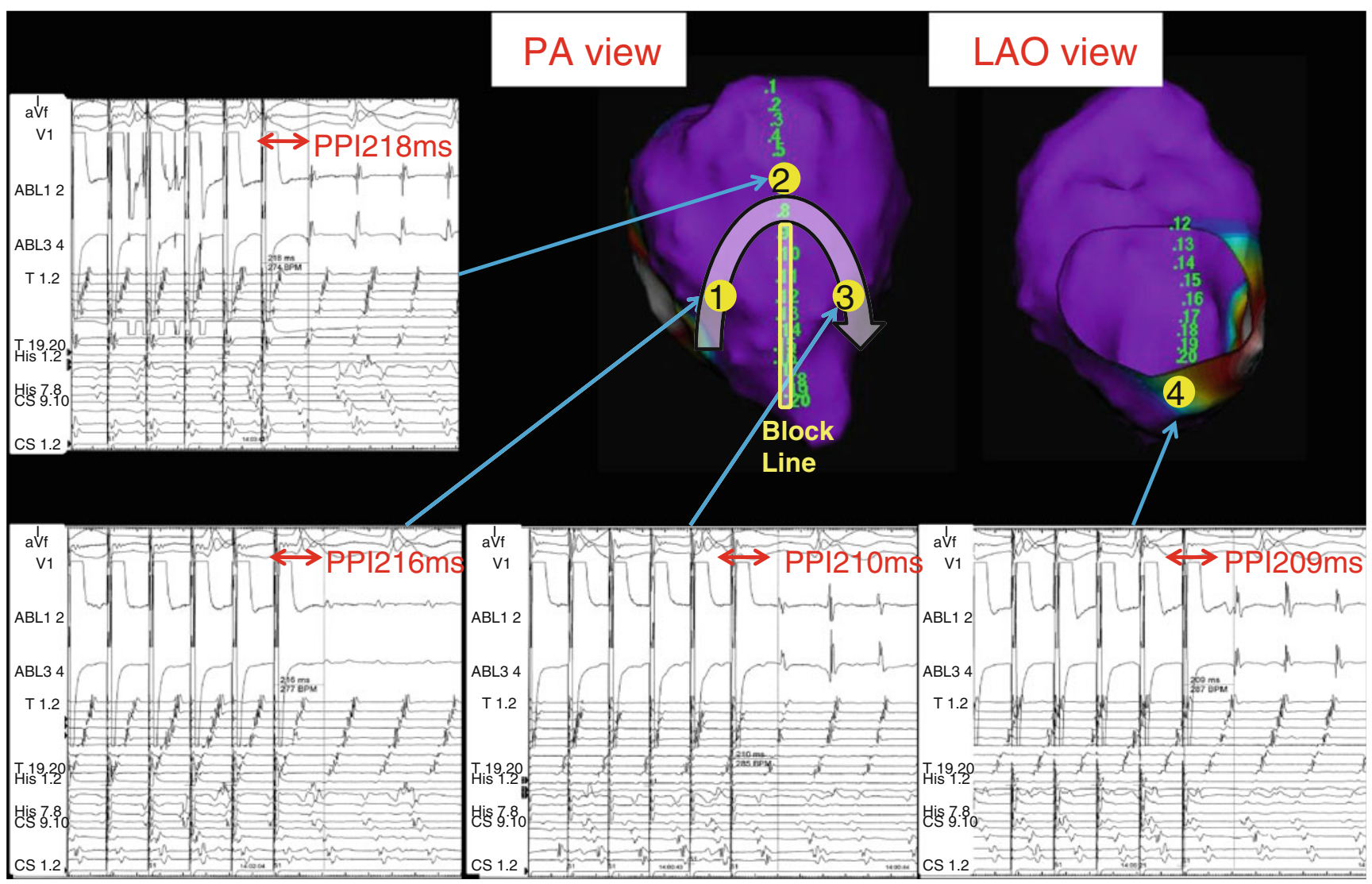

Fig. 5 Entrainment pacing during typical AFL (37-year-old female; CL $210 \mathrm{~ms}$, counterclockwise rotation). Entrainment pacing was performed around the block line (1: septal side of the block line, 2: site between the top of the block line and the superior vena cava, 3 : lateral side of the block line), and cavotricuspid isthmus (4). Cycle length $(C L)$ of the AFL was $210 \mathrm{~ms}$ and entrainment was performed at $190 \mathrm{~ms}$. The postpacing interval $(P P I)$ minus the CL of the AFL was

participation in the AFL circuit in about half the subjects. Our results also indicated that majority of the cases had incomplete transverse conduction at posterior RA during AFL. Other recent studies showed that the wavefront from the septal RA propagated to the lateral RA through a posterior RA $[19,20]$. On the other hand, Okumura et al. [21] described the noncontact mapping and entrainment mapping located in the upper turnaround point of the AFL activation in the anterior portion of the high RA at the SVC orifice in the majority of their patients. The discrepancy between their findings and ours might be explained by the color-range setting of the isopotential map and the analysis of the virtual unipolar morphologies in the posterior RA during AFL. We analyzed the many points of virtual unipolar electrograms which were independent of the color-range setting. On the other hand, Okumura et al. did not show the figure of the virtual unipolar electrograms of the posterior block line.
$<30 \mathrm{~ms}$ at all points. PA view posterior-anterior view, $L A O$ view left anterior oblique view. Intracardiac recordings from the mapping catheter (ABL1 and 2 are distal and ABL3 and 4 are proximal), distal to proximal Halo electrograms around TA (T1, 2 to T19, 20), catheter located at His bundle lesion, and proximal to the distal coronary sinus (CS9, 10 to CS1, 2)

\subsection{Clinical implications}

In patients with typical AFL, 3D noncontact mapping was not only a useful tool to determine the reentrant circuits but also a practical guide to recognize the turnaround point of the wavefront. Analysis of VUEM may also be useful for detecting the block lines and turnaround points of other arrhythmia circuits. In particular, when detailed contact mapping is not possible due to non-sustained or nontolerated arrhythmias, this method may be helpful for detecting the ablation point. Further studies of the VUEM of the ablation line are needed.

\section{Limitations}

One limitation of the present study is that we did not record the contact unipolar electrograms in all patients. In the first nine patients, we recorded the contact unipolar electrograms 
in the posterior RA during AFL and compared with the virtual unipolar electrogram. We found the accuracy of the virtual unipolar electrogram morphologies compared with contact unipolar electrogram morphologies in those patients. A previous study investigated the correlation between virtual and contact unipolar electrograms and reported that the accuracy of electrogram reconstruction was good at sites less than $40 \mathrm{~mm}$ from the balloon center [22]. In our study, the mean distance from the 20 points of the virtual unipolar electrograms to the MEA center was $24.5 \pm 2.2 \mathrm{~mm}$. We considered that there is a good correlation between the contact and noncontact unipolar electrograms in this study because the distance from the virtual unipolar points to the MEA center was very short.

\section{Conclusions}

The findings of the present study demonstrated that analysis of VUEM of noncontact mapping is a reliable method of identifying the posterior block line and its upper limit in patients with typical or reverse typical AFL. At the turnaround point of the wavefront, the second component of the VUEM changed from an $\mathrm{rS}$ to QS pattern. This method may be applied to detect the block line and its end in the circuits of other arrhythmias.

Open Access This article is distributed under the terms of the Creative Commons Attribution Noncommercial License which permits any noncommercial use, distribution, and reproduction in any medium, provided the original author(s) and source are credited.

\section{References}

1. Schumacher, B., Jung, W., Lewalter, T., \& Wolpert, C. (1999). Verification of linear lesions using a noncontact multielectrode array catheter versus conventional contact mapping technique. Journal of Cardiovascular Electrophysiology, 10, 791-798.

2. Schilling, R. J., Peters, N. S., Goldberger, J., Kadish, A. H., \& Davies, D. W. (2001). Characterization of the anatomy and conduction velocities of the human right atrial flutter circuit determined by noncontact mapping. Journal of the American College of Cardiology, 38, 385-393.

3. Friedman, P. A., Luria, D., Fenton, A. M., Munger, T. M., Jahangir, A., Shen, W. K., et al. (2000). Global right atrial mapping of human atrial flutter: The presence of posteromedial (sinus venosa region) functional block and double potentials. Circulation, 101, 1568-1577.

4. Fukuzawa, K., Yoshida, A., Kubo, S., Takano, T., Kiuchi, K., Kanda, G., et al. (2008). Upper turnover portion of the reentry circuit for typical and reverse typical atrial flutter. Pacing and Clinical Electrophysiology, 31, 1160-1167.

5. Fransisco, G., Cosio, F. G., Fernando, A., Barbero, J. M., Kallmeyer, C., \& Goicolea, A. (1988). Validation of double-spike electrograms as markers of conduction delay or block in atrial flutter. The American Journal of Cardiology, 61, 775-780.
6. Ishida, A., Yoshida, A., Kitamura, H., Kubo, S., Fukuzawa, K., \& Yamashiro, K. (2003). Identification of functional block line in atrial flutter using three-dimensional intracardiac echocardiography. Circulation Journal, 67, 940-944.

7. Shah, D., Haissaguerre, M., Takahashi, A., Jais, P., Hocini, M., \& Clementy, J. (2000). Differential pacing for distinguishing block from persistent conduction through an ablation line. Circulation, 102, 1517-1522.

8. Olshansky, B., Okumura, K., Henthorn, R. W., \& Wald, A. L. (1990). Characterization of double potentials in human atrial flutter: Studies during transient entrainment. Journal of the American College of Cardiology, 15, 833-841.

9. Okishige, K., Kawabata, M., Umayahara, S., Yamashiro, K., Gotoh, M., Isobe, M., et al. (2003). Radiofrequency catheter ablation of various kinds of arrhythmias guided by virtual electrograms using a noncontact, computerized mapping system. Circulation Journal, 67, 455-460.

10. Tai, C. T., Liu, T. Y., Lee, P. C., Lin, Y. J., Chang, M. S., \& Chen, S. A. (2004). Non-contact mapping to guide radiofrequency ablation of atypical right atrial flutter. Journal of the American College of Cardiology, 44, 1080-1086.

11. Zheng, L., Yao, Y., Zhang, S., Chen, W., Zhang, K., Wang, F., et al. (2009). Organized left atrial tachyarrhythmia during stepwise linear ablation for atrial fibrillation. Journal of Cardiovascular Electrophysiology, 20, 499-506.

12. Spach, M. S., Miller, W. T., Geselowitz, D. B., Barr, R. C., Kootsey, J. M., \& Johnson, E. A. (1981). The discontinuous nature of propagation in normal canine cardiac muscle. Circulation Research, 48, 39-54.

13. Spach, M. S., Miller, W. T., Miller-Jones, E., Warren, R. B., \& Barr, R. C. (1979). Extracellular potentials related to intracellular action potentials during impulse conduction in anisotropic canine cardiac muscle. Circulation Research, 45, 188-204.

14. Villacastin, J., Almendral, J., Arenal, A., Castellano, N. P., Gonzalez, S., Ortiz, M., et al. (2000). Usefulness of unipolar electrograms to detect isthmus block after radiofrequency ablation of typical atrial flutter. Circulation, 102, 3080-3085.

15. Lin, Y. J., Tai, C. T., Huang, J. L., Liu, T. Y., Lee, P. C., Ting, C. T., et al. (2004). Characteristics of virtual unipolar electrograms for detecting isthmus block during radiofrequency ablation of typical atrial flutter. Journal of the American College of Cardiology, 43, 2300-2304.

16. Sanchez-Quintana, D., Anderson, R. H., Cabrera, J. A., Climent, V., Martin, R., Farre, J., et al. (2002). The terminal crest: Morphological features relevant to electrophysiology. Heart, 88, 406-411.

17. Schalij, M. J., Boersma, L., Huijberts, M., \& Allessie, M. A. (2000). Anisotropic reentry in a perfused 2-dimensional layer of rabbit ventricular myocardium. Circulation, 102, 2650-2658.

18. Dixit, S., Lavi, N., Robinson, M., Riley, M. P., Callans, D. J., Marchlinski, F. E., et al. (2011). Noncontact electroanatomic mapping to characterize typical atrial flutter: Participation of right atrial posterior wall in the reentrant circuit. Journal of Cardiovascular Electrophysiology (in press)

19. Tai, C. T., Huang, J. L., Lee, P. C., Ding, Y. A., Chang, M. S., \& Chen, S. A. (2004). High-resolution mapping around the crista terminalis during typical atrial flutter: New insights into mechanisms. Journal of Cardiovascular Electrophysiology, 15, 406-414.

20. Tai, C. T., Ong, M. G., \& Chen, S. A. (2008). Electrophysiologic characteristics of the sinus venosa in patients with typical atrial flutter. Pacing and Clinical Electrophysiology, 31, 99-102.

21. Okumura, Y., Watanabe, I., Nakai, T., Ohkubo, K., Kofune, T., Ashino, S., et al. (2010). Upper turnaround point of the reentry circuit of common atrial flutter-Three-dimensional mapping and entrainment study. Journal of Interventional Cardiac Electrophysiology, 29, 147-156.

22. Kadish, A., Hauck, J., Pederson, B., Beatty, G., \& Gornick, C. (1999). Mapping of atrial activation with a noncontact, multielectrode catheter in dogs. Circulation, 99, 1906-1913. 